\title{
Fabrication, characterization and response surface method optimization for quantum efficiency of fluorescent nitrogen-doped carbon dots obtained from carboxymethylcellulose of oil palms empty fruit bunch
}

\begin{abstract}
Bio based nitrogen doped carbon dots (N-CDs) were obtained from empty fruit bunch carboxymethylcellulose and ethylenediamine (EDA) through one-pot hydrothermal carbonization route. The optimum as-formed N-CDs were thoroughly characterized via Transmission electron microscopy (TEM), high-resolution TEM (HRTEM), Fourier transform infrared (FTIR), X-ray photoelectron spectra (XPS), UV-vis spectra (UV-Vis) and Fluorescence spectra (PL). Response surface methodology was statistically used to assess three independent variables that have major influence on the fluorescence quantum yield (QY), including temperature $\left(230-270{ }^{\circ} \mathrm{C}\right)$, time $(2-$ $6 \mathrm{~h}$ ) and EDA mass $(10 \%-23.3 \%)$. Based on analysis of variance (ANOVA) results, synthesis temperature was found to be the most influential factor on the QY, followed by time and EDA mass. Higher temperature, long synthesis time and high amount of EDA were satisfactorily enough for efficient carbonization conversion rate and obtaining highest QY of N-CDs. The obtained quadratic model $(\mathrm{R} 2=0.9991)$ shows a good correlation between the experimental data and predicted values. The optimum synthetic parameters are of $270{ }^{\circ} \mathrm{C}$ temperature, $6 \mathrm{~h}$ reaction time and $23.3 \%$ of EDA mass. The optimized as-made N-CDs exhibited blue photoluminescence with both excitation dependent/independent phenomena and high nitrogen content. The maximum emission intensity was $426 \mathrm{~nm}$ at a maximum excitation wavelength of $320 \mathrm{~nm}$, with a QY of up to $22.9 \%$. XPS and FTIR data confirmed the existence of polar containing groups, such as carbonyl, carboxyl, hydroxyl and amino groups over the surface of N-CDs whereas nitrogen species in the form of (pyridinic and graphitic $-\mathrm{N}$ ) were introduced in the aromatic carbon domains, which imparts the hydrophilic and photostability of N-CDs. Taking into account the lowcost and sustainable production of N-CDs, this method considered a feasible route for converting low quality waste into value-added nanomaterials and utilizing for different functionalization processes and analytical applications.
\end{abstract}

Keyword: Carbon dots; Fluorescence; Response surface methodology; Optimization; Nitrogen doped 